\title{
Evaluation of the Anterior Chamber Angle in Angle- Closure Suspect Patients by Anterior Segment Optical Coherence Tomography (AS-OCT) and Pentacam
}

Mohamed Salah El-Din Mahmoud ( $\nabla$ drmsalah1982@yahoo.com )

Minia Faculty of medicine https://orcid.org/0000-0003-4537-2177

Nosiba Essam

Minia University Faculty of Medicine

Layla Mohamed Hammouda

Minia University Faculty of Medicine

Ahmed Shawkat Mohamed

Minia University Faculty of Medicine

\section{Research Article}

Keywords: Primary angle, closure suspect, Anterior segment optical coherence tomography, Anterior chamber angle, Pentacam

Posted Date: July 9th, 2021

DOI: https://doi.org/10.21203/rs.3.rs-684174/v1

License: (a) (i) This work is licensed under a Creative Commons Attribution 4.0 International License.

Read Full License 


\section{Abstract}

Purpose: The study aims is to detect primary angle-closure glaucoma (PACG) suspects using both anterior segment optical coherence tomography (AS-OCT) and Scheimpflug imaging.

Methods: A prospective cross-sectional observational study on forty PACG suspect patients with a range of ages from 20 to 70 years. Angle imaging by AS-OCT (Optovue Inc., Fairmont, CA) with study parameters in the superior, inferior, nasal, and temporal quadrants were anterior chamber angle (ACA), angle opening distance at $750 \mu \mathrm{m}$ (AOD750), and trabecular-iris space area at $750 \mu \mathrm{m}$ (TISA). Also, angle imaging using Pentacam (Oculus Optikgeräte $\mathrm{GmbH}$, Wetzlar, Germany) with study parameters were the anterior chamber angle (ACA), anterior chamber depth (ACD) and, anterior chamber volume (ACV). The angle evaluation was done before and after the mydriatic test.

Results: As regarding AS-OCT, after the mydriatic test, we noticed a decrease in all parameters measured, some decreased significantly while others did not. A significant decrease was noticed in ACA (TIA750) in all quadrants ( $P$ value $<0.001$ in nasal, 0.001 in superior \&temporal, 0.003 in inferior) and in TISA750 in the nasal quadrant only (P-value 0.004). AOD750 and TISA750 in the remaining quadrants also decreased but not significantly as ACA. As regarding Pentacam, all parameters decreased after the mydriatic test. ACA (P-value $<0.001)$ and ACD (P-value 0.004) showed significant statistical decrease but ACV showed statistically non-significant decrease (P-value 0.558 ).

Conclusions: AS-OCT and Pentacam are reliable investigations for the detection of PACG suspects which needs more close follow-up for a possible increase in IOP.

\section{Background:}

Glaucoma is an optic nerve disease of progressive course and it is one of the main diseases that can cause blindness worldwide. Fortunately, glaucoma can be avoided with prophylaxis, this makes early detection and therefore early treatment is critical ${ }^{(1)}$.

Glaucoma causes loss of the ganglion cells of the retina which is responsible for the changes seen in the optic nerve head, the defects present in the visual field (VF), and the loss in the nerve fiber layer of the retina. For VF to show a defect, at least $40 \%$ of RGCs have to be lost, therefore early diagnosis in the glaucoma suspect stage is very important ${ }^{(2)}$

Two types of glaucoma suspects are known: Primary Open-angle and primary angle-closure suspect ${ }^{(3)}$.

Primary angle-closure suspects can be recognized by gonioscopic visualization of the trabecular meshwork only in $180^{\circ}$ or less of the angle of the anterior chamber, but on condition that the intraocular pressure (IOP) is normal and the optic nerve head shows no structural damage ${ }^{(4)}$. 
Optical coherence tomography (OCT) was firstly designed to image the posterior segment and retina, but it was quickly adapted to visualize the anterior segment and cornea ${ }^{(5)}$.

Scheimpflug technique also offers a non-contact method for imaging the anterior segment. Although it is unable to show the angle effectively as AS-OCT, the anterior chamber volume measured by it is effective in screening for narrow angled patients ${ }^{(4)}$.

\section{Materials And Methods:}

A prospective cross-sectional observational study was conducted in Minia University hospital's ophthalmology department attending 40 PAC suspect patients between April 2019 and December 2019. Approval of the ethical committee of the faculty of medicine Minia University was obtained and informed written consent to be involved in the study was obtained from each patient.

Inclusion criteria: The patients' ages range from 20 to 70 years with shallow AC known by the Van Herrick test. Hyperopic refraction was done with Nidek® auto refractometer and with retinoscopy. With gonioscopy done with Goldmann 3 mirror lens (Ocular, USA): without indentation, there was an inability to visualize $\leq 180^{\circ}$ of the posterior trabecular meshwork (TM) in the absence of peripheral anterior synechia. IOP $\leq 21$ measured by Goldmann applanation tonometry (Keeler, UK). Normal optic nerve head $(\mathrm{ONH})$ appearance on fundus examination with both direct ophthalmoscope (Keeler, VISTA20, UK) and central lens of Goldmann 3 mirror lens (Ocular, USA). Normal visual field on static automated perimetry (SAP) testing using a $24-2$ protocol and a SITA-Standard strategy done with Humghery field analyzer (754i, Germany). Also, persons with a family history of primary angle-closure disease were included.

Exclusion criteria: Eyes with significant corneal opacity that can impair signal strings of any of the used imaging modalities were excluded. Also, eyes with a history of intraocular surgery (e.g. cataract surgery, retinal detachment surgery), a history of laser procedures (e.g. Laser peripheral iridotomy), eyes with anterior segment pathology as iridocyclitis or angle dysgenesis, eyes with a history of trauma, and eyes with findings suggestive of 2ry cause of glaucoma (e.g. iris or angle neovascularization, any iris or corneal abnormalities, dilated episcleral vessels) were excluded.

\section{Angle imaging by:}

1- Spectral-domain AS-OCT device, version 2015 (Optovue, Inc., Fremont, California) was done for each patient (both eyes) before and after mydriatic test with detection of the 3 measurements depending on the site of the scleral spur, AC angle (trabecular iris angle at $750 \mu \mathrm{m}$ ), angle opening distance at $750 \mu \mathrm{m}$ from the scleral spur and trabecular-iris space area at $750 \mu \mathrm{m}$ from the scleral spur, and represented by the mean values of the nasal, temporal, superior and inferior values.(Fig. 1,2)

The mydriatic test: 
A mydriatic agent used was a combination of $50 \mathrm{mg}$ cyclopentolate $\mathrm{HCL}$ and $500 \mathrm{mg}$ phenylephrine $\mathrm{HCL}$, it was instilled in both eyes. AS-OCT\&Pentacam imaging was done before and after cyclopherine ED installation by 30 minutes when the pupil becomes mid dilated as this allows for maximal crowding of the iris in the angle, (pupil block was sufficient to allow the pressure in the posterior chamber to 'push' the iris on to the cornea).

2- Pentacam:(Oculus Optikgeräte GmbH, Wetzlar, Germany) was done for each patient (both eyes) before and after the mydriatic test.

Each Scheimpflug image was analyzed for anterior chamber angle (ACA) which is the angle between the cornea and the root of the iris, anterior chamber depth (ACD) which is the axial distance between the corneal endothelium and the anterior surface of the crystalline lens and anterior chamber volume (ACV) which is calculated with Pentacam system using Wang formula. (Fig. 3,4)

\section{Statistical analysis}

A statistical study using SPSS version 25. Descriptive statistics were done for parametric quantitative data by mean \pm standard deviation, and non-parametric quantitative data by the median and interquartile range (IQR), while they were done for categorical data by number and percentage. Distribution of the data was done by the Shpiro Wilk test. Analyses were done for non-parametric quantitative data using Wilcoxon signed-rank test between the two times. The level of significance was taken at $(P$-value $<0.05)$.

\section{Results:}

The mean age of the patients was $46 \pm 10.2$ years (range: 25 to 65 years). Twenty-eight patients (70\%) were females and twelve patients $(30 \%)$ were males. Patients with a positive family history of angleclosure glaucoma were nine (22.5\%) while thirty-one patients (77.5 \%) were negative for family history.

\section{1-AS-OCT parameters:}

\section{1-In the superior quadrant (Table 1)}

A-ACA (TIA750): There was a high statistically significant difference between its values before and after the mydriatic test, (P-value: 0.001), the median before the mydriatic test was $30.29 *(I O R: 26-35)$, while the median after the mydriatic test was $25^{*}$ (IQR: 22-33).

B-AOD750: There was a statistically non-significant difference between AOD750 values before and after the mydriatic test, (P-value 0.361), as its median before the mydriatic test was $482 \mathrm{Mm}$ (IQR: 402.75555.5), while its median after the mydriatic test was 424 Mm (IQR: 365.25-600.5).

C-TISA750: There was a statistically non-significant difference between TISA750 values before and after the mydriatic test (P-value 0.194), as its median before the mydriatic test was $0.25 \mathrm{~mm}^{2}$ (IQR: 0.21-0.29), and after the mydriatic test, it was $0.22 \mathrm{~mm}^{2}$ (IQR: 0.18-0.3). (Table 1). 
Table (1): Showing the difference in the values of anterior chamber angle parameters in superior quadrant before and after the mydriatic test:

\begin{tabular}{|lllllr|}
\hline & $\begin{array}{l}\text { Median before } \\
\text { mydriasis }\end{array}$ & $\begin{array}{l}\text { Median after } \\
\text { mydriasis }\end{array}$ & $\begin{array}{l}\text { IQR before } \\
\text { mydriasis }\end{array}$ & $\begin{array}{l}\text { IQR after } \\
\text { mydriasis }\end{array}$ & $\begin{array}{l}\mathrm{P}- \\
\text { value }\end{array}$ \\
\hline ACA(TIA750) & $30.29^{*}$ & $25^{\star}$ & $26-35^{\star}$ & $22^{\star}-33^{*}$ & 0.001 \\
\hline AOD750 & $482 \mathrm{Mm}$ & $424 \mathrm{Mm}$ & $\begin{array}{l}402.75 \mathrm{Mm}- \\
555.5 \mathrm{Mm}\end{array}$ & $\begin{array}{l}365.25 \mathrm{Mm}- \\
600.5 \mathrm{Mm}\end{array}$ & 0.361 \\
\hline TISA750 & $0.25 \mathrm{~mm}^{2}$ & $0.22 \mathrm{~mm}^{2}$ & $0.21 \mathrm{~mm}^{2}-0.29 \mathrm{~mm}^{2}$ & $0.18 \mathrm{~mm}^{2}-0.3 \mathrm{~mm}^{2}$ & 0.194 \\
\hline
\end{tabular}

\section{2-In the inferior quadrant (Table 2)}

A-ACA (TIA750): There was a high statistically significant difference between ACA (TIA750) values before and after the mydriatic test, (P-value: 0.003$)$, the median before the mydriatic was $32.93 *(I O R$ : 27.69-37), while the median after mydriatic was $27 *$ (IQR: 22.36-33).

B- AOD750: There was a statistically non-significant difference between AOD750 values before and after the mydriatic test, (P-value 0.803), as its median before mydriasis was $517 \mathrm{Mm}$ (IQR: 443.5-666), while its median after mydriasis was 485 Mm (IQR: 398-732).

C- TISA750: There was a statistically non-significant difference between TISA750 values before and after the mydriatic test (P-value 0.306 ), as its median before the mydriatic test was $0.28 \mathrm{~mm}^{2}$ (IQR: $0.24-0.33$ $\mathrm{mm}^{2}$ ), and after the mydriatic test it was $0.26 \mathrm{~mm}^{2}$ (IQR: 0.21-0.33). (Table 2).

Table (2): Showing the difference in the values of anterior chamber angle parameters in inferior quadrant before and after the mydriatic test:

\begin{tabular}{|llllll|}
\hline & $\begin{array}{l}\text { Median before } \\
\text { mydriasis }\end{array}$ & $\begin{array}{l}\text { Median after } \\
\text { mydriasis }\end{array}$ & $\begin{array}{l}\text { IQR before } \\
\text { mydriasis }\end{array}$ & $\begin{array}{l}\text { IQR after } \\
\text { mydriasis }\end{array}$ & $\begin{array}{l}\text { P- } \\
\text { value }\end{array}$ \\
\hline ACA(TIA750) & $32.93^{*}$ & $27^{*}$ & $27.69-37$ & $22.36-33$ & 0.003 \\
\hline AOD750 & $517 \mathrm{Mm}$ & $485 \mathrm{Mm}$ & $398-732$ & $398-732$ & 0.803 \\
\hline TISA750 & $0.28 \mathrm{~mm}^{2}$ & $0.26 \mathrm{~mm}^{2}$ & $0.24-0.3$ & $0.21-0.33$ & 0.306 \\
\hline
\end{tabular}

\section{3-In the nasal quadrant (Table 3)}

A-ACA (TIA750): There was a high statistically significant difference between ACA (TIA750) values before and after the mydriatic test, (P-value: $<0.001)$, the median before mydriatic was $34 *(I O R: 29-37)$ while median after mydriatic was 27* (IQR: 23.1-35.75). 
B- AOD750: There was a statistically non-significant difference between its values before and after the mydriatic test, (P-value 0.341), as its median before the mydriatic test was $562.5 \mathrm{Mm}$ (IQR: 470-653.5), while its median after the mydriatic test was 485 Mm (IQR: 405.25-684.5).

C- TISA750: There was a statistically highly significant difference between TISA750 values before and after the mydriatic test (P-value $\mathbf{0 . 0 0 4}$ ), as its median before the mydriatic test was $0.29 \mathrm{~mm}^{2}$ (IQR: $0.25-$ $0.36 \mathrm{~mm}^{2}$ ), and after the mydriatic test it was $0.26 \mathrm{~mm}^{2}$ (IQR: 0.22-0.33). (Table 3).

Table (3): Showing difference in the values of anterior chamber angle parameters in nasal quadrant before and after mydriatic test:

\begin{tabular}{|llllll|}
\hline & $\begin{array}{l}\text { Median before } \\
\text { mydriasis }\end{array}$ & $\begin{array}{l}\text { Median after } \\
\text { mydriasis }\end{array}$ & $\begin{array}{l}\text { IQR before } \\
\text { mydriasis }\end{array}$ & $\begin{array}{l}\text { IQR after } \\
\text { mydriasis }\end{array}$ & $\begin{array}{l}\text { P- } \\
\text { value }\end{array}$ \\
\hline ACA(TIA750) & $34^{*}$ & $27^{*}$ & $29-37$ & $23.1-35.75$ & $<$ \\
\hline AOD750 & $562.5 \mathrm{Mm}$ & $485 \mathrm{Mm}$ & $470-653.5$ & $405.25-684.5$ & 0.341 \\
\hline TISA750 & $0.29 \mathrm{~mm} 2$ & $0.26 \mathrm{~mm} 2$ & $0.25-0.36$ & $0.22-0.33$ & 0.004 \\
\hline
\end{tabular}

\section{4- In the temporal quadrant: ( Table 4)}

A-ACA (TIA750): There was a high statistically significant difference between ACA (TIA750) values before and after the mydriatic test, (P-value: <0.001), the median before the mydriatic test was 34*(IOR: 26.3341) while the median after the mydriatic test was $28 *$ (IQR: 22.81-36).

B-AOD750: There was a statistically non-significant difference between AOD750 values before and after the mydriatic test, (P-value0.951), as its median before the mydriatic test was $546 \mathrm{Mm}$ (IQR: 392.5-750), while it's median after the mydriatic test was 539 Mm (IQR: 421-702.25).

C-TISA750: There was a statistically non-significant difference between TISA750 values before and after the mydriatic test (P-value $\mathbf{0 . 1 5 3 )}$, as its median before the mydriatic test was $0.3 \mathrm{~mm}^{2}$ (IQR: $0.22-0.37$ ), and after the mydriatic test it was $0.27 \mathrm{~mm}^{2}$ (IQR: 0.21-0.35). (Table 4).

Table (4): Showing the difference in the values of anterior chamber angle parameters in the temporal quadrant before and after the mydriatic test: 


\begin{tabular}{|llllll|}
\hline & $\begin{array}{l}\text { Median before } \\
\text { mydriasis }\end{array}$ & $\begin{array}{l}\text { Median after } \\
\text { mydriasis }\end{array}$ & $\begin{array}{l}\text { IQR before } \\
\text { mydriasis }\end{array}$ & $\begin{array}{l}\text { IQR after } \\
\text { mydriasis }\end{array}$ & $\begin{array}{l}\text { P- } \\
\text { value }\end{array}$ \\
\hline ACA(TIA750) & $34^{*}$ & $28^{*}$ & $26.33-41$ & $22.81-36$ & 0.001 \\
\hline AOD750 & $546 \mathrm{Mm}$ & $539 \mathrm{Mm}$ & $392.5-750)$, & $421-702.25$ & 0.951 \\
\hline TISA750 & $0.3 \mathrm{~mm}^{2}$ & $0.27 \mathrm{~mm}^{2}$ & $0.22-0.37$ & $0.21-0.35$ & 0.153 \\
\hline
\end{tabular}

\section{2-Pentacam parameters (Table 5)}

A-ACA: there was a high statistically significant difference between ACA values before and after the mydriatic test (in the form of a decrease in ACA measurements after mydriasis i.e. narrowing of the angle), (P-value: $<0.001)$, the median before the mydriatic test was $33^{*}\left(\right.$ IOR: $\left.30^{*}-37.98^{*}\right)$ while the median after the mydriatic test was $29 *($ IQR: $23.2 *-34.38 *)$.

B-ACD: there was a statistically highly significant difference between ACD values before and after the mydriatic test (in the form of a decrease in ACD measurements after mydriasis i.e. decrease in the depth of AC), (P-value 0.004), as its median before the mydriatic test was $2.59 \mathrm{~mm}$ (IQR: $2.34 \mathrm{~mm}-2.75 \mathrm{~mm}$ ), while it's median after the mydriatic test was $2.45 \mathrm{~mm}$ (IQR: $2.11 \mathrm{~mm}-2.7 \mathrm{~mm}$ ).

C-ACV: there was a statistically non-significant difference between ACV values before and after the mydriatic test (P-value 0.558 ), as its median before the mydriatic test was $135 \mathrm{~mm}^{3}$ (IQR: $100.75 \mathrm{~mm}^{3}$ $165.75 \mathrm{~mm}^{3}$ ), and after the mydriatic test it was $128.5 \mathrm{~mm}^{3}$ (IQR: $105.25 \mathrm{~mm}^{3}-164.5 \mathrm{~mm}^{3}$ ). (Table 5).

Table (5): Showing the difference in the values of anterior chamber angle parameters with Pentacam before and after the mydriatic test:

\begin{tabular}{|llllll|}
\hline & $\begin{array}{l}\text { Median } \\
\text { before } \\
\text { mydriasis }\end{array}$ & $\begin{array}{l}\text { Median after } \\
\text { mydriasis }\end{array}$ & IQR before mydriasis & IQR after mydriasis & $\begin{array}{l}\text { P- } \\
\text { value }\end{array}$ \\
\hline ACA & $33^{*}$ & $29^{*}$ & $30^{*}-37.98^{*}$ & $23.2^{*}-34.38^{*}$ & $<$ \\
\hline ACD & $2.59 \mathrm{~mm}$ & $2.45 \mathrm{~mm}$ & $2.34 \mathrm{~mm}-2.75 \mathrm{~mm}$ & $2.11 \mathrm{~mm}-2.7 \mathrm{~mm}$ & 0.004 \\
\hline ACV & $135 \mathrm{~mm}^{3}$ & $128.5 \mathrm{~mm}^{3}$ & $100.75 \mathrm{~mm}^{3}-165.75 \mathrm{~mm}^{3}$ & $105.25 \mathrm{~mm}^{3}-164.5 \mathrm{~mm}^{3}$ & 0.558 \\
\hline
\end{tabular}

\section{Discussion:}

Angle-closure glaucoma is one of the sight-threatening diseases and is a major cause of worldwide blindness ${ }^{(6)}$. 
The reversible attachment of the trabecular meshwork to the periphery of the iris is defined as appositional angle-closure. It occurs due to physiological movement of the pupil and can lead to transient IOP increase in narrow angled eyes. If it became chronic, angle configuration will show permanent changes that will lead to increases in IOP and finally will cause PACG ${ }^{(7)}$.

A relationship was found between the configuration of the anterior chamber and primary angle-closure (PAC) ${ }^{(8)}$ it was proved that shallow anterior chamber and narrow-angle were significant risk factors for PACG, so, measurement of IOP and evaluation of anterior chamber angle are important for the prevention and early treatment of PACG ${ }^{(9)}$.

Anterior chamber assessment can be done by multiple methods as Van Herrick technique, gonioscopy, ultrasound biomicroscopy (UBM), and AS-OCT and Scheimpflug photography ${ }^{(8)}$.

As regarding the Van Herrick technique, it is simple and doesn't need direct contact with the ocular surface, but it is highly subjective ${ }^{(9)}$. Gonioscopy is considered the standard method for angle examination, but it has some problems that limit its use in assessing anterior chamber angle status. Firstly, it requires a certain degree of skill and experience to give trustable results. Secondly, any increase in illumination or pressure on the lens can change the findings. Lastly, it is considered to some degree a subjective technique ${ }^{(10)}$.

Unfortunately, although UBM is very helpful in examination of anterior chamber angle, it has some difficulties as the need to place the patient in the supine position and to apply a topical anaesthetic and an ocular cup to the eye also,it requires skill and is equipment dependent, thus, it is not suitable for screening for narrow angled eyes ${ }^{(11)}$. Now, AS-OCT allows cross-sectional imaging of the anterior chamber and the angle with high-resolution. Also, it doesn't require contact with the eye and provides rapid examination. Another advantage is that the patients are examined while they are sitting. Also, it has good repeatability and reproducibility ${ }^{(12)}$.

Recently, the Pentacam-Scheimpflug camera has been started to be used in the imaging of the anterior chamber angle. It is a non-contact method and gives high-resolution images. It creates 3-dimensional images of the anterior eye segment. The Pentacam parameters that are useful in glaucoma are ACD, ACV, corneal thickness (apical), and ACA, as well as inbuilt IOP correction formulae. Despite these advantages, it is not efficient as anterior segment OCT in the evaluation of the anterior chamber angle due to the inability to visualize the sclera spur. However, it is better than AS-OCT in giving more quantitative data of the anterior chamber, due to measuring peripheral ACD and ACV. Therefore, it has a promising future as a screening method for detecting narrow angled eyes ${ }^{(13)}$.

This present study was conducted in Minia University Hospital Ophthalmology Department between April 2019 and December 2019, on forty PACS patients. We had chosen patients between twenty and seventy years age group who were either hyperopes, with a positive family history of angle-closure glaucoma, 
with shallow AC, or with gonioscopy, 180 degrees of their trabecular meshwork could not be seen, provided that their IOP is less than $21 \mathrm{mmHg}$, no optic neuropathy and their visual field test was normal.

Patients who gave history of intraocular surgery or laser procedures to the eye, who gave a history of trauma, those with corneal opacity or anterior segment pathology or had any cause of 2dry glaucoma as angle neovascularization were excluded from this study.

In all patients, we examined different parameters of the anterior chamber using both AS-OCT and Pentacam before and after installation of one drop of combination of $5 \mathrm{mg}$ cyclopentolate HCL and $500 \mathrm{mg}$ phenylephrine HCL by 30 minutes.

AS regarding AS-OCT we measured ACA (TIA750), AOD750, and TISA750 in superior, inferior, nasal and temporal quadrants. After the mydriatic test, we noticed a decrease in all parameters measured, some decreased significantly while others did not. A significant decrease was noticed in ACA (TIA750) in all quadrants (P-value $<0.001$ in nasal, 0.001 in superior \&temporal, 0.003in inferior) and in TISA750 in the nasal quadrant only (P-value 0.004). AOD750 and TISA750 in the remaining quadrants also decreased but not significantly as ACA.

As regarding Pentacam, we measured $A C A, A C D$, and ACV before and after the mydriatic test. All parameters were noticed to be decreased after the mydriatic test. ACA (P-value < 0.001) and ACD ( $P$-value $0.004)$ showed significant statistical decrease but ACV showed statistically non-significant decrease (Pvalue 0.558 ).

The results we had obtained, revealed a decrease in all parameters of the anterior chamber measured by both AS-OCT\&Pentacam after the mydriatic test was done.

We also measured IOP before and after the mydriatic test. There was a significant increase in its measurements after pupillary dilatation (P-value $<0.001)$.

These changes that occurred in the parameters of anterior chamber and IOP after the mydriatic test are mostly attributed to the effect of the mydriatic agent which induces appositional angle-closure by one of these mechanisms of PACG:

(1) Pupillary block type: when the pupil becomes mid dilated the iris becomes crowded in the angle, (this is because the resulting block of the pupil causes the aqueous in the posterior chamber to push the iris towards the cornea so that, the angle is narrowed and the aqueous drainage is hindered and IOP becomes elevated). This effect is exaggerated in susceptible eyes (with narrow angles).

(2) Plateau iris type: when the pupil dilates in the eyes in which the iris is plateau shaped the iris becomes crowded in the angle causing trabecular meshwork occlusion.

(3) Lens factors: the cycloplegic agent present in the cyclopherine ED produces relaxation of ciliary muscle making the lens more spherical causing some decrease in ACD (14). 
In this study, the main mechanism of angle narrowing in PACS eyes is mostly the pupillary block one

In a study done by Aptel et al ${ }^{(15)}$., with shifting from light to dark(i.e. with physiologic mydriasis), angle opening distance (AOD) at $500 \mu \mathrm{m}$ decreased significantly more in fellow eyes of acute angle-closure patients than in PACS while the mean iris volume increased significantly in the fellow eyes of these patients and decreased significantly in most PACS eye, and in all open-angle eyes. Unlike our study, they used physiologic dilatation instead of pharmacologic one. It matches ours in that AOD 500 decreased in PACS after physiologic mydriasis. (But in ours theAOD750 decrease is insignificant).

In a study done by Yamada et al ${ }^{(7)}$ on seventy eyes of seventy consecutive patients with primary angleclosure suspect, primary angle-closure, or primary angle-closure glaucoma (based on gonioscopy), ASOCT and IOP measurement was done for all those patients after the mydriatic provocative test (MPT) and the dark room prone provocative test (DRPPT), their results revealed that ACD and AOD 500 of the positive group(with a significant increase in IOP after the two tests), using the MPT, were significantly less than those of the negative group (with an insignificant increase in IOP). The trabecular-iris space area 500 of the positive group was significantly less than the negative group, using both the MPT and the DRPPT. This agrees with our study in the significant IOP elevation in PACS group after mydriatic test and in the decrease of both AOD \&TISA after mydriatic test, but we differ from them in measuring AOD \&TISA at $750 \mathrm{Mm}$ from the scleral spur not at $500 \mathrm{Mm}$ and the decrease in both of them in our study is insignificant. We also differ from them in measuring ACD with Pentacam (not with AS-OCT), but both studies showed a significant decrease in ACD after the mydriatic test.

In another study done also by Aptel et al ${ }^{(16)}$ included the fellow eyes of patients who suffered from a previous acute attack of primary angle-closure glaucoma and eyes of control subjects with open angles. All patients were imaged by AS-OCT after phenylephrine $10 \%$ instillation and another time after tropicamide $1 \%$ instillation. The axial length and depth of anterior chamber did not change significantly after mydriasis. Also, in the eyes of the control group, the mean AOD 500 and TISA 500 did not change significantly after pupil dilation, while both of them were about 2 to 3 times smaller after mydriasis in the eyes of the case group, regardless of the mydriatic agent used. These results are consistent with our study which showed a decrease in AOD750 and TISA750 after mydriatic installation (but the decrease in ours is insignificant). We differ from them in measuring ACD with Pentacam not by AS-OCT and our study showed significant decrease in its value after mydriasis, unlike this study which showed nonsignificant change.

There is a study done by Hirose et al ${ }^{(17)}$ showed that in the angle-closure group in each of the four quadrants, the iris thickness (IT) increased significantly in the dark, and the AOD500 and TISA500 decreased significantly in the dark. This is mostly due to physiologic dilatation of the pupil in the darkness which induces relative pupillary block and thickening of the iris periphery leading to narrowing in the angle of the anterior chamber, therefore decrease in AOD and TISA. This decrease in AOD and TISA after physiologic mydriasis is consistent with our study which revealed a decrease in these two 
parameters after pharmacologic mydriasis (but our decrease was insignificant in four quadrants except TISA in nasal quadrant).

Similarly, in a study done by Masoodi et al, ACA and AOD500 in the nasal and temporal quadrants were significantly greater in light compared to dark (all with $p=0.000$ ). This is also consistent with our study (18).

Also, an old previous study was done by Ishikawa et al ${ }^{(19)}$, under light conditions and with using UBM all eyes tested showed open angles. On the other hand, on darkroom provocation, the angles of $55.6 \%$ of the eyes were occluded and those of $44.4 \%$ of the eyes were not. In the dark, evidence of appositional angleclosure was found in the angles in 99 patients (55.6\%) during testing. The eyes in which the angles were occluded showed decrease in ARA and AOD500 in the dark (this is consistent also with our study).

Another idea was done by Kobayashia et al ${ }^{(20)}$, who studied the effect of miosis (using pilocarpine) on anterior chamber in narrow angled patients. After pilocarpine instillation, the whole narrow angled eyes showed an increase in the TIA, AOD250, and AOD500. Also, they noticed that pilocarpine induced a decrease in ACD which was more in eyes with narrow angles than in those with wide angles. (This is consistent with our study in the effect over TIA and AOD but does not match ours in the effect on ACD).

Another study consistent with ours, the one done with Friedman et al ${ }^{(21)}$. it revealed that in both angleclosure cases and controls the AOD500\&750 and the angle-recess area showed significant decrease in the dark using UBM (the decrease was more in cases than in controls).(This is consistent with our study). With Scheimpflug Camera there was noticed decrease (of borderline statistical significance) in the average angle width in the dark, (it was more in cases than in controls). (This also agrees with our study in a decrease of $A C D$ and ACV post mydriatic). On the other hand, with instillation of pilocarpine, both cases and controls showed a significant increase in AOD $500 \& 750 \mu \mathrm{m}$ and angle-recess area with UBM. (This increase was less in cases than in controls) (These results agree with our study).

Leung et al ${ }^{(22)}, \mathbf{K}$ woo et al ${ }^{(23)}$,Jouzdani et al ${ }^{(24)}$, and Ming Young et al ${ }^{(25)}$., also found the same results in the dark.

In another study done by Razeghinejad et al, ${ }^{(26)}$ revealed that both mydriatic test and water drinking test performed in PACS patients caused a significant increase in IOP. This agrees with our study in significant IOP elevation after the mydriatic test.

Lavanya et al ${ }^{(27)}$ also, agreed with us in post mydriatic increase in IOP in narrow-angle patients.

The results in all the studies that agree with us in the decrease in parameters of AC and increase in IOP after mydriasis (physiologic or pharmacologic) are mostly attributed to the same mechanisms of PACG we mentioned before (especially the pupillary block). 
On the other hand, a study was done by Jing-Min Guo et al ${ }^{(28)}$, found that iris thickness at $750 \mu \mathrm{m}$, in addition to depth and volume of the anterior chamber had increased significantly after pharmacologic mydriasis, while iris curve, cross-sectional area and volume had decreased significantly in both PACS and normal controls. This study differs from ours in that post mydriatic the AOD500 increased none significantly while in ours AOD750 decreased none significantly. Also, the significant increase in ACD and ACV in PACS after mydriasis does not match with our study which revealed a significant decrease in ACD and a non-significant decrease in ACV after mydriasis.

Also, another study was done by Razeghinejad et al ${ }^{(29)}$ showed that the mydriatic test didn't cause a significant difference in intraocular pressure or parameters of anterior chamber except the anterior chamber volume, which increased after the mydriatic test. Our study differs from this one in many aspects, firstly, in ours, there was a significant increase in IOP measurements after mydriasis, secondly, this study revealed a significant increase in ACV post mydriatic while ours showed a non-significant decrease in ACV post mydriatic

The difference in techniques and ethnicities of persons included in the previous studies can explain the discrepancies in their results.

Our study had some limitations. First, its results cannot be generalized to larger populations because it wasn't a population-based study but a hospital-based one. Also, we did not use UBM for the precise diagnosis of plateau iris configuration. Although some studies used AS-OCT to detect plateau iris configuration, its definitive diagnosis should be done with UBM.

\section{Declarations:}

\section{Availability of data and materials}

The data in the present study are available from the corresponding authors upon reasonable request.

\section{Acknowledgements}

None.

\section{Funding}

None

\section{Author notes}

All authors contributed equally to this work.

\section{Ethics declarations}

\section{Ethics approval and consent to participate}


This study was approved by the Ethics Committee of the faculty of medicine of Minia University with approval No.225:5/2019. Written informed consent was provided by all subjects before the study.

\section{Competing interests}

The authors declare that they have no competing interests.

\section{References:}

1. Almasieh M, Levin LA (2017). Neuroprotection in glaucoma: Animal models and clinical trials. Annu Rev Vis Sci.; 3: 91-120.

2. Deshpande GA, Bawankule PK, Raje DV, Chakraborty M (2019). Linear discriminant score for differentiating early primary open angle glaucoma from glaucoma suspects. Indian $\mathrm{J}$ Ophthalmol.;67:75-81.

3. Vijaya L, Asokan R, Panday M, George R (2017). Is prophylactic laser peripheral iridotomy for primary angle-closure suspects a risk factor for cataract progression? The Chennai Eye Disease Incidence Study. Br J Ophthalmol.;101: 665-670.

4. Thomas R, Parikh R, Muliyil J, Kumar RS (2003). Five-year risk of progression of primary angleclosure to primary angle-closure glaucoma: a population-based study. Acta Ophthalmol Scand;81:480-5.

5. Grewal DS, Tanna AP. Diagnosis of glaucoma and detection of glaucoma progression using spectral domain optical coherence tomography. Curr Opin Ophthalmol. 2013; 24: 150-161.

6. Petsas, Anna, George Chapman, and Richard Stewart (2017) "Acute angle-closure glaucoma-A potential blind spot in critical care." Journal of the Intensive Care Society.;18.3: 244-246.

7. Yamada, R., Hirose, F., Matsuki, T., Kameda, T., \& Kurimoto, Y (2016). Comparison of mydriatic provocative and dark room prone provocative tests for anterior chamber angle configuration. Journal of Glaucoma.; 25(6), 482-486

8. Priya L, Dabasia B, Edgar DF, Lawrenson JG (2013). Methods of measurement of the anterior chamber angle Part 1: Angle-closure glaucoma and gonioscopy. Optometry in Practice.;14(3):107117. .

9. Sun X, Dai Y, Chen Y, Yu DY, Cringle SJ, Chen J, Kong X, Wang X, Jiang C (2017). Primary angleclosure glaucoma: what we know and what we don't know. Progress in retinal and eye research.;57, 26-45.

10. Park, S. B., Sung, K. R., Kang, S. Y., Jo, J. W., Lee, K. S., \& Kook, M. S (2011). Assessment of narrow angles by gonioscopy, Van Herick method and anterior segment optical coherence tomography. Japanese journal of ophthalmology. 55(4), 343-350.

11. Angmo, D., Nongpiur, M. E., Sharma, R., Sidhu, T., Sihota, R., \& Dada, T (2016).Clinical utility of anterior segment swept-source optical coherence tomography in glaucoma. Oman journal of ophthalmology. 9(1), 3. 
12. Sharma R, Sharma A, Arora T, Sharma S, Sobti A, Jha B, Chaturvedi N, Dada T (2014). Application of anterior segment optical coherence tomography in glaucoma. Survey of ophthalmology; 59(3), 311327.

13. Raluca, M., Mircea, F., Andrei, F., Carmen, D., Miruna, N., Grigorios, T., \& lleana, U (2015). Old and new in exploring the anterior chamber angle. Romanian journal of ophthalmology. 59(4), 208.

14. Lavanya R, Baskaran M, Kumar RS, Wong HT, Chew PT, Foster PJ, Friedman DS, Aung T (2012). Risk of acute angle-closure and changes in intraocular pressure after pupillary dilation in Asian subjects with narrow angles. Ophthalmology. 119(3), 474-480.

15. Aptel, F., Chiquet, C., Beccat, S., \& Denis, P (2012). Biometric evaluation of anterior chamber changes after physiologic pupil dilation using Pentacam and anterior segment optical coherence tomography. Investigative ophthalmology \& visual science. 53(7), 4005-4010.

16. Aptel, F., \& Denis, P (2011). Optical coherence tomography quantitative analysis of iris volume changes after pharmacologic mydriasis. Ophthalmology.2011;117(1), 3-10.

17. Hirose, F., Hata, M., Ito, S. I., Matsuki, T., \& Kurimoto, Y (2013). Light-dark changes in iris thickness and anterior chamber angle width in eyes with occludable angles. Graefe's Archive for Clinical and Experimental Ophthalmology; 251(10), 2395-2402.

18. Masoodi, H., Jafarzadehpur, E., Esmaeili, A., Abolbashari, F., \& Hosseini, S. M. A (2014). Evaluation of anterior chamber angle under dark and light conditions in angle-closure glaucoma: an anterior segment OCT study. Contact Lens and Anterior Eye; 37(4), 300-304.

19. Ishikawa, H., Esaki, K., Liebmann, J. M., Uji, Y., \& Ritch, R (1999). Ultrasound Biomicroscopy Dark Room Provocative Testing: A Quantitative Method for Estimating Anterior Chamber Angle Width. Japanese journal of ophthalmology; 43(6), 526-534.

20. Kobayashi, H., Kobayashi, K., Kiryu, J., \& Kondo, T (1999). Pilocarpine induces an increase in the anterior chamber angular width in eyes with narrow angles. British journal of ophthalmology; 83(5), 553-558.

21. Friedman DS, Gazzard G, Foster P, Devereux J, Broman A, Quigley H, Tielsch J, Seah S (2003). Ultrasonographic biomicroscopy, Scheimpflug photography, and novel provocative tests in contralateral eyes of Chinese patients initially seen with acute angle-closure. Archives of ophthalmology; 121(5), 633-642.

22. Leung, C. K. S., Cheung, C. Y. L., Li, H., Dorairaj, S., Yiu, C. K. F., Wong, A. L., ... \& Lam, D. S. C (2007). Dynamic analysis of dark-light changes of the anterior chamber angle with anterior segment OCT. Investigative ophthalmology \& visual science; 48(9), 4116-4122.

23. Woo, E. K., Pavlin, C. J., Slomovic, A., Taback, N., \& Buys, Y. M (1999). Ultrasound biomicroscopic quantitative analysis of light-dark changes associated with pupillary block. American journal of ophthalmology; 127(1), 43-47.

24. Jouzdani, S., Amini, R., \& Barocas, V. H (2019).Contribution of different anatomical and physiologic factors to iris contour and anterior chamber angle changes during pupil dilation: theoretical analysis. Investigative ophthalmology \& visual science;54(4), 2977-2984. 
25. Young, S. M., Aquino, M. C., Shabana, N., Zheng, C., Loon, S. C., See, J. L., ... \& Chew, P. T (2014). Ultrasound biomicroscopic comparison of primary open-angle glaucoma and primary angle-closure glaucoma eyes in dark and light conditions. Asian Journal of Ophthalmology; 14(1), 5-16.

26. Razeghinejad, R., \& Nowroozzadeh, M. H (2019) . Water-drinking Test and Pharmacologic Mydriasis as Provocative Tests in Primary Angle-closure Suspects. Journal of Ophthalmic \& Vision Research;14(3), 267.

27. Lavanya R, Baskaran M, Kumar RS, Wong HT, Chew PT, Foster PJ, Friedman DS, Aung T (2012). Risk of acute angle-closure and changes in intraocular pressure after pupillary dilation in Asian subjects with narrow angles. Ophthalmology; 119(3), 474-480.

28. Guo, J. M., Li, M., Xu, X. L., Zhang, H., \& Wang, J. M (2015) Anterior segment changes after pharmacologic mydriasis using Pentacam and optical coherence tomography in angle-closure suspects. International journal of ophthalmology; 8(5), 980.

29. Razeghinejad, M. R., Lashkarizadeh, H., Nowroozzadeh, M. H., \& Yazdanmehr, M (2016). Changes in ocular biometry and anterior chamber parameters after pharmacologic mydriasis and peripheral iridotomy in primary angle-closure suspects. Journal of optometry; 9(3), 189-19.

\section{Figures}

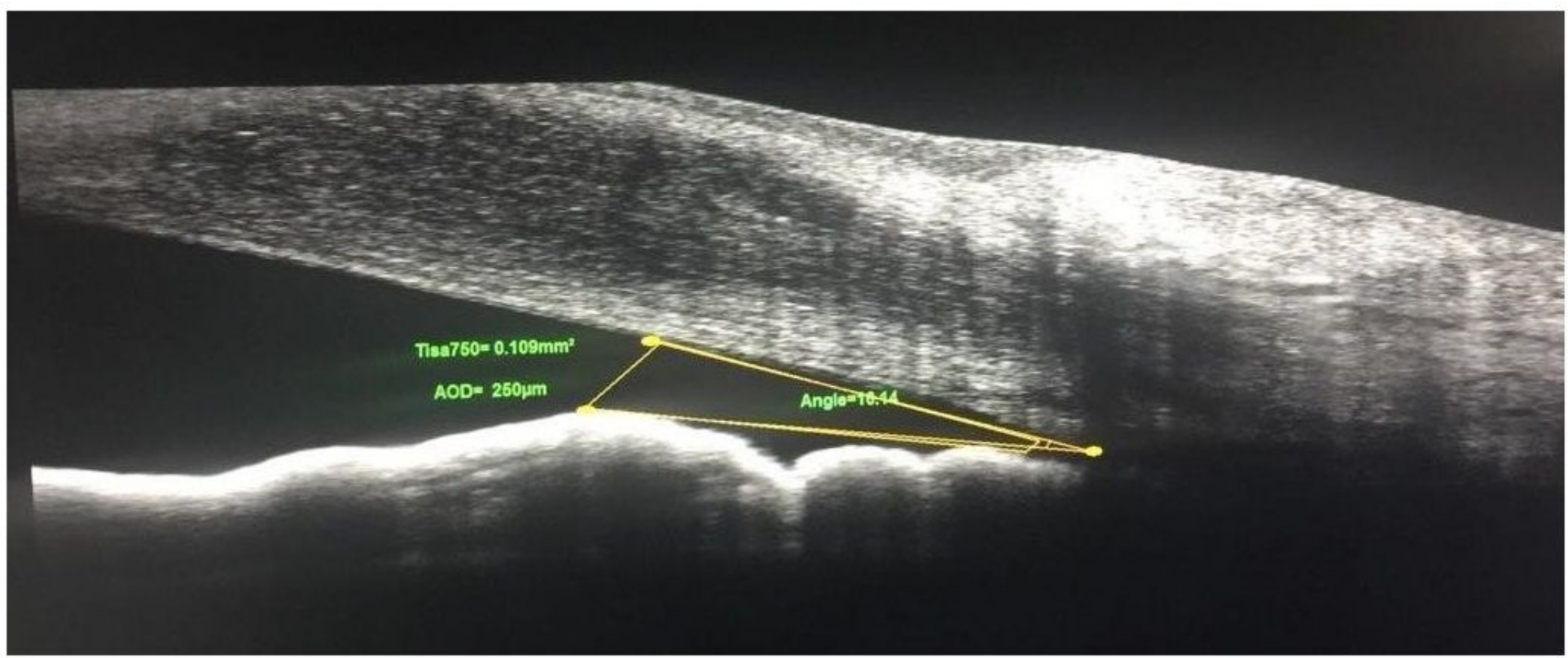

Figure 1

AS-OCT printout of the anterior chamber angle in nasal quadrant of the right eye before mydriasis. 


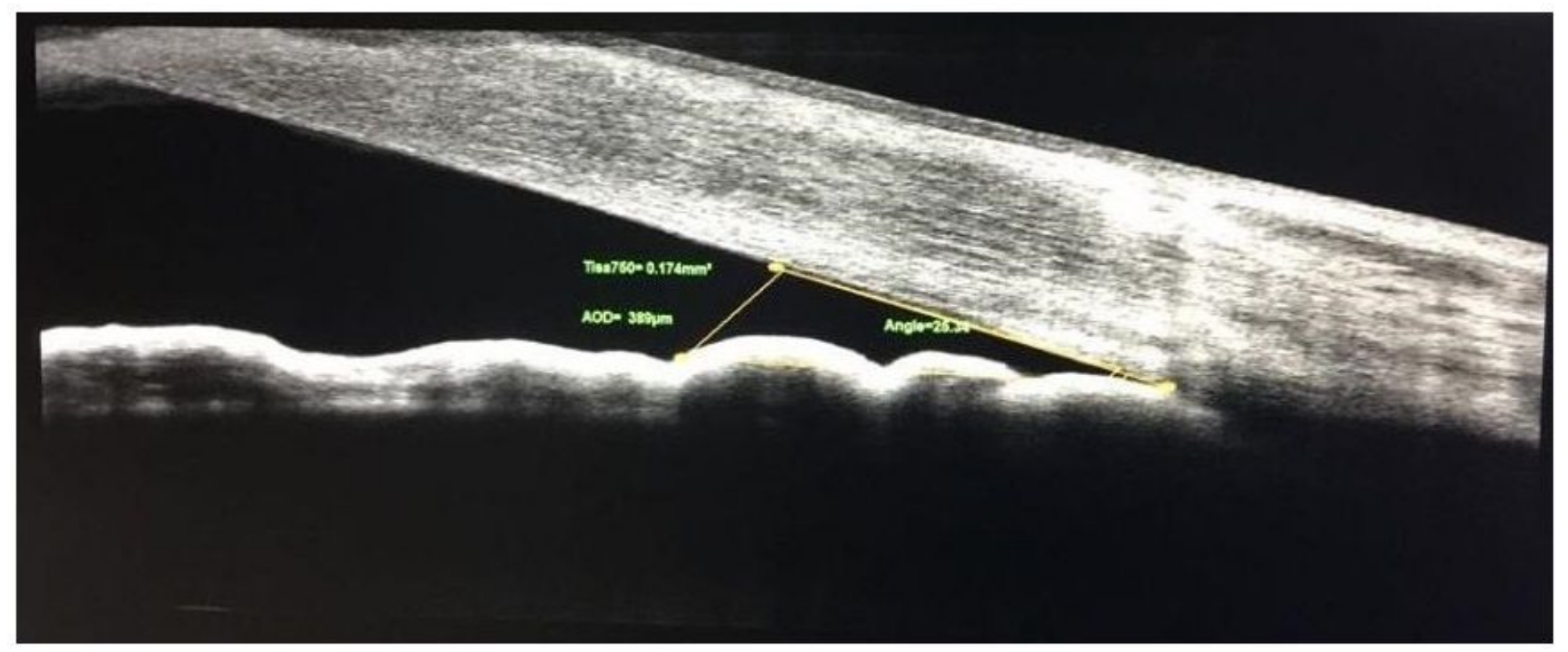

Figure 2

AS-OCT printout of the anterior chamber angle in the same quadrant of the same eye but after mydriasis. 


\section{OCULUS - PENTACAM Overview}
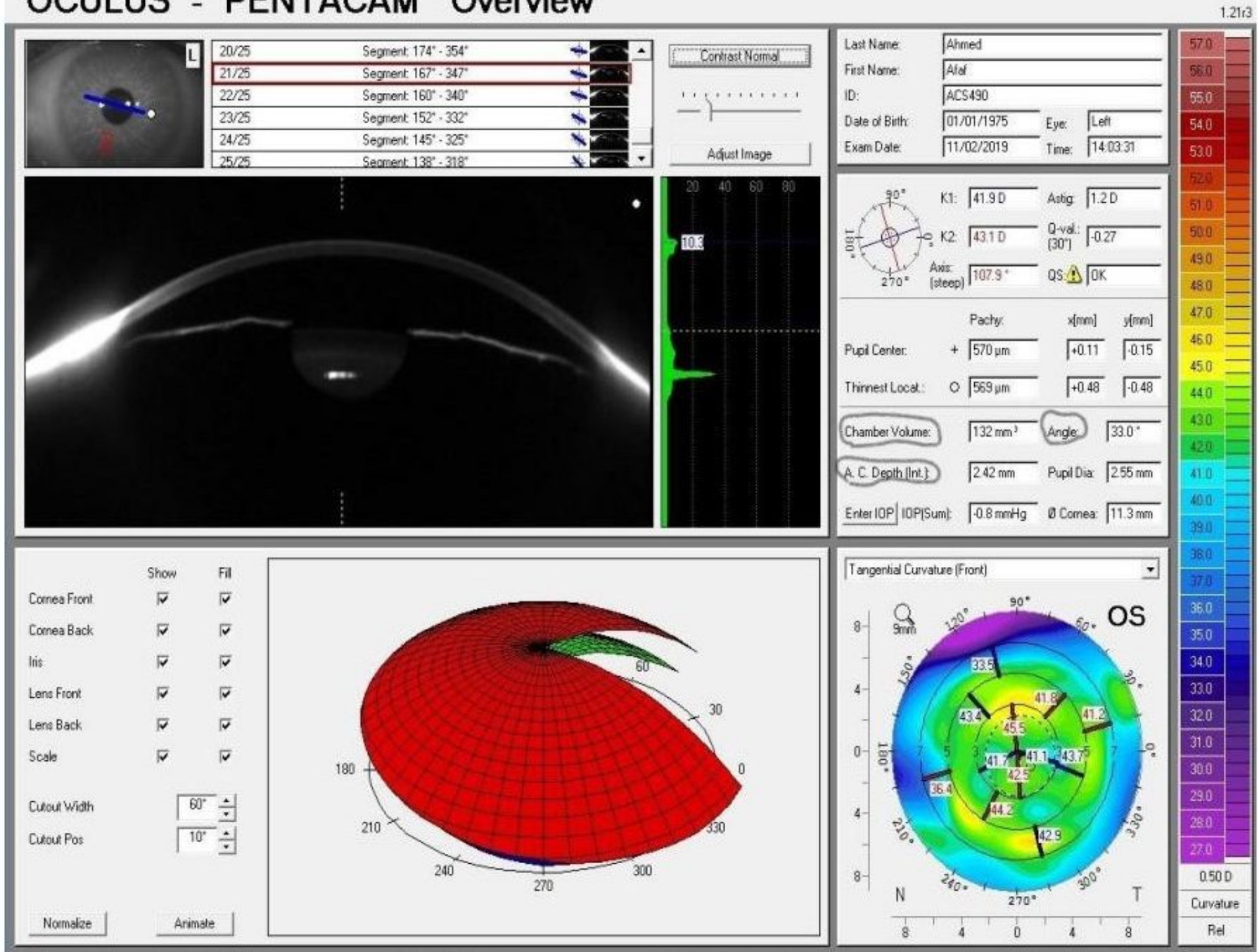

Oculus Optikgerăte $\mathrm{GmbH}$

35582 Wetzlar

Tel. (0641) 20 05-0

Münchholzhäuser Str. 29

Fax (0641) 2005-255

monvoculus de

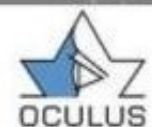

Figure 3

Scheimpflug image display of the left eye before mydriasis. 


\section{OCULUS - PENTACAM Overview}

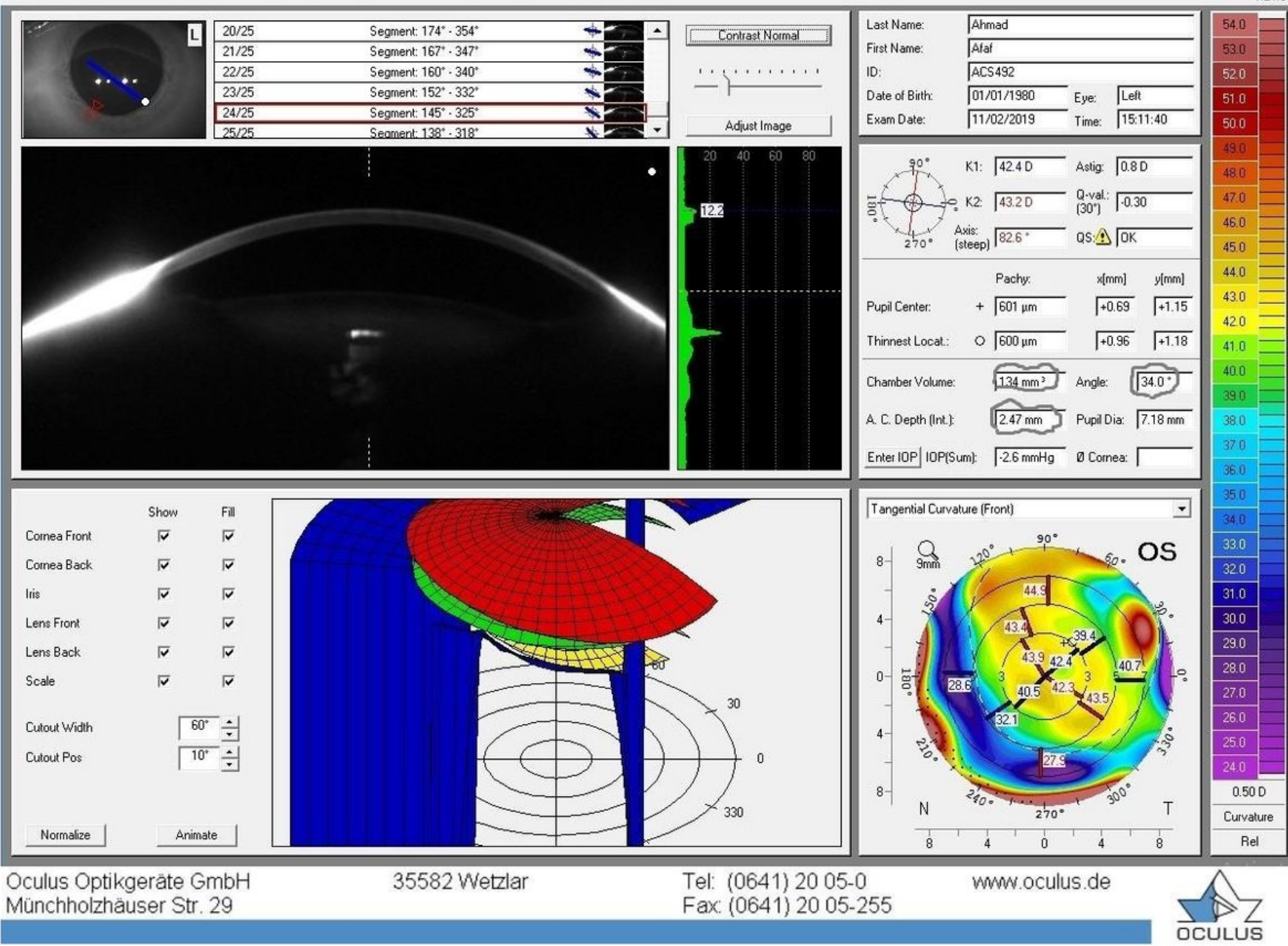

\section{Figure 4}

Scheimpflug image display of the same eye in the same patient but after mydriasis. 